\title{
The General Two Dimensional Shifted Jacobi Matrix Method for Solving the Second Order Linear Partial Difference-differential Equations with Variable Coefficients
}

\author{
Z. Kalateh Bojdi ${ }^{1}$, S. Ahmadi-Asl ${ }^{1}$, A. Aminataei ${ }^{2, *}$ \\ ${ }^{1}$ Department of Mathematics, Birjand University, Birjand, Iran \\ ${ }^{2}$ Faculty of Mathematics, K. N. Toosi University of Technology, P.O. Box 16315-1618, Tehran, Iran \\ ${ }^{*}$ Corresponding Author: ataei@kntu.ac.ir
}

Copyright (C)2013 Horizon Research Publishing All rights reserved.

\begin{abstract}
In this paper, a new and efficient approach for numerical approximation of second order linear partial differential-difference equations (PDDEs) with variable coefficients is introduced. Explicit formulae which express the two dimensional Jacobi expansion coefficients for the derivatives and moments of any differentiable function in terms of the original expansion coefficients of the function itself are given in the matrix form. The main importance of this scheme is that using this approach reduces solving the general linear PDDEs to solve a system of linear algebraic equations, wherein greatly simplify the problem. In addition, some experiments are given to demonstrate the validity and applicability of the method.
\end{abstract}

Keywords General Two Dimensional Jacobi Matrix Method, Second Order Linear Pddes with Variable Coefficients

2010 AMS subject classifications: $35-X X, 33 C 45$

\section{Introduction}

PDDEs arise in many branches of science and technology. For instance, in electromagnetic theory, physics, elasticity, fluid mechanics, heat transfer, acoustics, quantum mechanics, and so on [1-15]. In physics for example, the heat flow and the wave propagation phenomena are well described by PDDEs [5]. In ecology, most population models are governed by PDDEs [5]. The dispersion of a chemically reactive material is characterized by PDDEs [5]. In addition, most physical phenomena of fluid dynamics, quantum mechanics, electricity, plasma physics, propagation of shallow water waves, and many other models are controlled within its domain of validity by PDDEs [5]. Also the essential behavior of most physical systems can be modeled by the second order linear PDDEs with variable coefficients. There is no general theory known concerning the solvability of all PDDEs. Such a theory is extremely unlikely to exist, given the rich variety of physical, geometric, and probabilistic phenomena which can be modeled with PDDEs [2]. Instead research focuses on various particular PDDEs that are important for applications within and outside of mathematics, with the hope that insight from the origins of these PDDEs can give clues as to their solutions [2]. In particular case, the second order linear PDDEs with variable coefficients and their solutions play a major role in the branch of modern mathematics and arise frequently in many applied areas. Therefore, a reliable and efficient technique for the solution of them is too important. For a review of some applications of the second order linear PDDEs with variable coefficients, one can see [1-16]. The analytic results on the existence and uniqueness of solutions to the second order linear PDDEs have been investigated by many authors [1-16], however the existence and uniqueness of the solution for PDDEs under their conditions is beyond the scope of this article. We assume that the PDDEs which we consider in this paper with their conditions have solutions. During the last decades, several methods have been used to solve the second order linear PDDEs such as Chebyshev and Taylor method [17, 18], Adomian's decomposition method [19, 20], the differential transform method [21-23], the finite element method [24-27], the finite volume method [28], the spectral element method [28], and the Jacobi operational matrix method [29], using generalized Jacobi polynomials/functions [30]. In this paper, we develop a new and efficient scheme (the general two dimensional shifted Jacobi matrix method) for numerical 
approximation of the second order linear PDDE (with the initial and boundary conditions which has a solution which is expressible as a known elementary function) in the following form

$$
\begin{aligned}
& \sum_{\substack{j=0 \\
k}}^{1} \sum_{i=0}^{1} P_{i, j}(x, y) \frac{\partial^{i+j}}{\partial x^{i} \partial y^{j}} u(x+k, y+h)=g(x, y), a \leq x \leq b, a \leq y \leq b, \\
& k, h \in \mathbb{Z},
\end{aligned}
$$

where the coefficients $P_{k, j}(x)$ and $g(x, y)$ have the two dimensional Taylor series expansions and $\mathbb{Z}$ denotes the set of integer numbers. The main importance of our work is considering the general second order linear PDDE (1), wherein the other papers only considered particular cases of our general problem. Also using the general shifted Jacobi polynomials as the basic functions for numerical approximation wherein the shifted Chebyshev (first to fourth kinds) and Legendre polynomials are particular cases of them, is the other superiority of our paper. The remainder of this paper is organized as follows: In Section 2, we introduce the properties of the one and two dimensional shifted Jacobi polynomials and the basic formulation of them is required for our subsequent development. Section 3 , is devoted to the operational matrix of the one and two dimensional shifted Jacobi polynomials (derivative and moment) with some useful theorems. Section 4, summarizes the application of the two dimensional shifted Jacobi matrix method to the solution of problem (1). Thus, a set of algebraic equations is formed and a solution of the considered problem is introduced. In Section 5, we present the approximation with Jacobi polynomials. In section 6 , the proposed method is applied to the two numerical experiments. The applications of the new method for the high order linear PDDEs is presented in Section 7. Finally, we have monitored a brief conclusion in section 8. Note, that we have computed the numerical results by Matlab (version 2013) programming.

\subsection{Preliminaries and notations}

We first introduce some notations, which we use in the following.

Definition 1. A PDDE is a relation that involves partial derivatives of an unknown function. Let the unknown function be $u$, and $x, y, \ldots$ be independent variables. i.e., $u=u(x, y, z, \ldots$,$) . Often, one of these variables represents$ the time. Thus a PDDE is an equation of the form

$$
F\left(x+h, y+k, z, u, u_{x}, u_{y}, u_{z}, u_{x x}, u_{x y}, \ldots, u_{x x x}, \ldots\right)=0,
$$

where $h$ and $k \in \mathbb{Z}$. In Eq.(2), we have used the subscript notation for the partial differentiation

$$
u_{x}=\frac{\partial u}{\partial x}, u_{x y}=\frac{\partial^{2} u}{\partial x \partial y},
$$

and so on. We will always assume that the unknown function $u$ is sufficiently well behaved so that all necessary partial derivatives exist and the corresponding mixed partial derivatives are equal, e.g.,

$$
u_{x y}=u_{y x}, u_{x z x}=u_{x x z},
$$

and so on.

Definition 2. As in the case of ordinary differential equations (ODEs), the order of the PDDEs is defined in Eq.(2), to be the highest order of partial derivatives appearing in the equation.

Definition 3. We say that the $\operatorname{PDDE}(2)$, is linear if $F$ is linear as a function of the variables $u_{x}, u_{y}, u_{z}, u_{x x}, \ldots$, . i.e., $F$ is a linear combination of the unknown function and its derivatives. Eq.(2) is said to be quasilinear if $F$ is linear as a function of the highest-order derivatives.

\section{The one and two dimensional shifted Jacobi polynomials}

The classical Jacobi polynomial of degree $n, J_{n}^{(\alpha, \beta)}(z)$ [31-33] is defined on the interval [-1,1] and can be determined with the aid of the following recurrence formulae:

$$
\begin{aligned}
& J_{0}^{(\alpha, \beta)}(z)=1, J_{1}^{(\alpha, \beta)}(z)=\frac{1}{2}(\alpha+\beta+2)+\frac{1}{2}(\alpha+\beta) \\
& J_{n+1}^{(\alpha, \beta)}(z)=\left(a_{n} z-b_{n}\right) J_{n}^{(\alpha, \beta)}(z)-c_{n} J_{n-1}^{(\alpha, \beta)}(z), \quad n \geq 1
\end{aligned}
$$

where

$$
\begin{aligned}
& a_{n}=\frac{(2 n+\alpha+\beta+1)(2 n+\alpha+\beta+2)}{2(n+1)(n+\alpha+\beta+1)}, \\
& b_{n}=\frac{(2 n+\alpha+\beta+1)\left(\beta^{2}-\alpha^{2}\right)}{2(n+1)(n+\alpha+\beta+1)(2 n+\alpha+\beta)},
\end{aligned}
$$

and

$$
c_{n}=\frac{(2 n+\alpha+\beta+2)(n+\beta)}{(n+1)(n+\alpha+\beta+1)(2 n+\alpha+\beta)} .
$$


For using the general shifted Jacobi polynomials on the interval $[a, b]$, we shift the defining domain by means of the following substitution:

$$
z=\frac{2 x-a-b}{b-a}, \quad a \leq x \leq b
$$

These polynomials construct the orthogonal polynomials with respect to the weight function $w^{(\alpha, \beta)}(x)=(b-x)^{\alpha}(x-a)^{\beta}$, over $[a, b]$

$$
\int_{a}^{b} P_{m}^{(\alpha, \beta)}(x) P_{n}^{(\alpha, \beta)}(x) w^{(\alpha, \beta)}(x) d x=\eta_{n}^{\alpha, \beta} \delta_{m n}
$$

where $\delta_{m n}$ is the Kronecker delta function and

$$
\eta_{n}^{\alpha, \beta}=\left\|P_{m}^{(\alpha, \beta)}(x)\right\|_{w^{(\alpha, \beta)}}^{2}=\frac{(b-a)^{\alpha+\beta+2 m-1} 2^{\alpha+\beta+2} \Gamma(m+\alpha+1) \Gamma(m+\beta+1)}{m !(2 m+\alpha+\beta+1) \Gamma(m+\beta+\alpha+1)} .
$$

A function $y(x) \in L_{w^{(\alpha, \beta)}}^{2}[a, b]$, can be expressed in terms of the general shifted Jacobi polynomials as

$$
y(x)=\sum_{i=0}^{\infty} a_{i} P_{i}^{(\alpha, \beta)}(x)
$$

where the coefficient $a_{i}$ is given by

$$
a_{i}=\frac{1}{\eta_{i}^{\alpha, \beta}} \int_{a}^{b} P_{i}^{(\alpha, \beta)}(x) y(x) w^{(\alpha, \beta)}(x) d x .
$$

In practice, only the first $m+1$ terms of the shifted Jacobi polynomials are considered. Then we have

$$
y_{m}(x)=\sum_{i=0}^{m} a_{i} P_{i}^{(\alpha, \beta)}(x)=\left(P^{(\alpha, \beta)}(x)\right)^{T} A,
$$

where the shifted Jacobi coefficient vector $A$ and the shifted Jacobi vector $P^{(\alpha, \beta)}(x)$ are given by

$$
A=\left[a_{0}, a_{1}, \ldots, a_{m}\right]^{T}
$$

and

$$
P^{(\alpha, \beta)}(x)=\left[P_{0}^{(\alpha, \beta)}(x), P_{1}^{(\alpha, \beta)}(x), \ldots, P_{m}^{(\alpha, \beta)}(x)\right]^{T}
$$

Similarly a function $f(x, t)$ of the two independent variables is defined on $a \leq x, y \leq b$ may be expanded in terms of the two dimensional shifted Jacobi polynomials as

$$
f_{m}(x, t)=\sum_{i=0}^{m} \sum_{j=0}^{m} c_{i, j} P_{i}^{(\alpha, \beta)}(x) P_{j}^{(\alpha, \beta)}(y)=\left(P^{(\alpha, \beta)}(x)\right)^{T} C\left(P^{(\alpha, \beta)}(y)\right),
$$

where the shifted Jacobi coefficients matrix $C$ is given by

$$
C=\left(\begin{array}{ccc}
c_{11} & \cdots & c_{1 m} \\
\vdots & \ddots & \vdots \\
c_{m 1} & \cdots & c_{m m}
\end{array}\right)
$$

where

$$
c_{i, j}=\frac{1}{\eta_{i}^{(\alpha, \beta)} \eta_{j}^{(\alpha, \beta)}} \int_{a}^{b} \int_{a}^{b} f(x, y) P_{i}^{(\alpha, \beta)}(x) P_{j}^{(\alpha, \beta)}(y) w^{\alpha, \beta}(x) w^{\alpha, \beta}(y) d x d y, i, j=1, \ldots, m .
$$

Now in the following theorem, we present the explicit form of the shifted Jacobi polynomials.

Theorem 1. The explicit form of the shifted Jacobi polynomials are in the following form

$$
P_{n}^{(\alpha, \beta)}(x)=\sum_{i=0}^{n} G_{i}^{(\alpha, \beta, n)} x^{i} ; \alpha, \beta>-1
$$

where

$$
G_{i}^{(\alpha, \beta, n)}=\sum_{k=i}^{n} \frac{2^{k}}{(b-a)^{k}}(-b)^{i-k} B_{k}^{(\alpha, \beta, n)}
$$

and

$$
B_{k}^{(\alpha, \beta, n)}=2^{-k}\left(\begin{array}{c}
n+\alpha+\beta+k \\
k
\end{array}\right)\left(\begin{array}{l}
n+\alpha \\
n-k
\end{array}\right) ; k=0,1,2, \ldots, n .
$$


Proof. From [34], we have

$$
J_{n}^{(\alpha, \beta)}(z)=\sum_{i=0}^{n} B_{i}^{(\alpha, \beta, n)}(1-z)^{i} ; \alpha, \beta>-1,
$$

where

$$
B_{k}^{(\alpha, \beta, n)}=2^{-k}\left(\begin{array}{c}
n+\alpha+\beta+k \\
k
\end{array}\right)\left(\begin{array}{l}
n+\alpha \\
n-k
\end{array}\right) ; k=0,1,2, \ldots, n .
$$

Now using substitution of Eq.(8) into Eq.(22) yields

$$
\begin{aligned}
& P_{n}^{(\alpha, \beta)}(x)=J_{n}^{(\alpha, \beta)}\left(\frac{2 x-a-b}{b-a}\right)=\sum_{i=0}^{n} B_{i}^{(\alpha, \beta, n)}\left(\frac{2}{b-a}\right)^{i}(x-b)^{i}= \\
& \sum_{i=0}^{n} B_{i}^{(\alpha, \beta, n)} \frac{2^{i}}{(b-a)^{i}} \sum_{k=0}^{i}\left(\begin{array}{l}
i \\
k
\end{array}\right)(-b)^{i-k} x^{k},
\end{aligned}
$$

from Eq.(24) and defining

$$
G_{i}^{(\alpha, \beta, n)}=\sum_{k=i}^{n} \frac{2^{k}}{(b-a)^{k}}(-b)^{i-k} B_{k}^{(\alpha, \beta, n)}
$$

the proof is completed.

Also from theorem 1, we can obtain the explicit form of the two dimensional shifted Jacobi polynomials as the following

$$
P_{n}^{(\alpha, \beta)}(x) P_{m}^{(\alpha, \beta)}(y)=\sum_{i=0}^{n} \sum_{j=0}^{m} G_{i}^{(\alpha, \beta, n)} G_{j}^{(\alpha, \beta, m)} x^{i} y^{j},
$$

and from the two dimensional Taylor series, we can obtain the following formula

$$
G_{i}^{(\alpha, \beta, n)} G_{j}^{(\alpha, \beta, m)}=\frac{\partial^{i+j} f(x, y)}{\partial x^{i} \partial y^{j}}(0,0),
$$

where

$$
f(x, y)=P_{n}^{(\alpha, \beta)}(x) P_{m}^{(\alpha, \beta)}(y) .
$$

From theorem 1, we can obtain the matrix relation between general shifted Jacobi polynomials space (set) $\left\{P_{0}^{(\alpha, \beta)}(x), P_{1}^{(\alpha, \beta)}(x), \ldots, P_{n}^{(\alpha, \beta)}(x)\right\}$ and standard polynomial space (set) as the following

$$
\left[P_{0}^{(\alpha, \beta)}(x), P_{1}^{(\alpha, \beta)}(x), \ldots, P_{n}^{(\alpha, \beta)}(x)\right]^{T}=K\left[1, x, \ldots, x^{n}\right]^{T}
$$

where $K$ is a lower triangular matrix

$$
(K)_{i, j}=\left\{\begin{array}{l}
0, \quad i>j, \\
G_{j}^{(\alpha, \beta, i)}, \quad i \leq j .
\end{array}\right.
$$

Theorem 2. The matrix $K$ is invertible if and only if $\alpha, \beta>0$, which is a consequence of the fact that the matrix associted to a change of bases is always nonsingular.

Proof: For establishing the invertibility of matrix $K$, it is sufficient to show that

$$
\operatorname{Det}(\mathrm{K}) \neq 0 \text {, }
$$

where $\operatorname{Det}(\mathrm{K})$ is a determinant of the square matrix $K$. But because $K$ is a lower triangular matrix, then we have

$$
\operatorname{Det}(K)=\prod_{i=0}^{n} G_{i}^{(\alpha, \beta, i)}
$$

and from (25) and (30), we have

$$
\begin{aligned}
& G_{i}^{(\alpha, \beta, i)}=\sum_{k=i}^{i} \frac{2^{k}}{(b-a)^{k}}(-b)^{i-k} B_{k}^{(\alpha, \beta, n)}= \\
& \frac{2^{i}}{(b-a)^{i}}(-b)^{i-i} B_{i}^{(\alpha, \beta, i)}=\frac{2^{i}}{(b-a)^{i}} 2^{-i}\left(\begin{array}{c}
2 i+\alpha+\beta \\
i
\end{array}\right)\left(\begin{array}{c}
i+\alpha \\
0
\end{array}\right)= \\
& \frac{\left(\begin{array}{c}
2 i+\alpha+\beta \\
i
\end{array}\right)}{(b-a)^{i}} \neq 0, \quad \forall \alpha, \beta>0,
\end{aligned}
$$

therefore the proof is completed. 
Remark 1. From theorem 2, we conclude that in situations that we need the invertibility of matrix $K$, choosing parameters $-1<\alpha, \beta<0$, is not suitable.

Now from (29), we can obtain the following important matrix relation, which is an immediate consequence of the above fact

$$
\left[1, x, \ldots, x^{m}\right]^{T}=K^{-1}\left[P_{0}^{(\alpha, \beta)}(x), P_{1}^{(\alpha, \beta)}(x), \ldots, P_{m}^{(\alpha, \beta)}(x)\right]^{T}, \forall \alpha, \beta>0
$$

\section{Operational matrices of the two dimensional shifted Jacobi polyno- mials (derivative, moment and shift)}

In this section, we present the operational matrices of the general shifted Jacobi polynomials (derivative, shift and moment). The derivative and moment operational matrices with respect to the classical Jacobi polynomials are obtaind in [35] and we present them in the following theorems.

To do this, first we introduce the concept of operational matrix.

\subsection{The operational matrix}

Definition 4. Suppose

$$
\phi=\left[\phi_{0}, \phi_{1}, \ldots, \phi_{n}\right]
$$

where $\phi_{0}, \phi_{1}, \ldots, \phi_{n}$ are the basis functions on the given interval $[a, b]$. The matrices $E_{n \times n}$ and $F_{n \times n}$ are named as the operational matrices of the derivatives and integrals respectively if and only if

$$
\begin{gathered}
\frac{\mathrm{d}}{\mathrm{dt}} \phi(t) \simeq E \phi(t), \\
\int_{a}^{x} \phi(t) d t \simeq F \phi(t) .
\end{gathered}
$$

Further assume $g=\left[g_{0}, g_{1}, \ldots, g_{n}\right]$, named as the operational matrix of the product, if and only if

$$
\phi(x) \phi^{T}(x) \simeq G_{g} \phi(x) .
$$

In other words, to obtain the operational matrix of a product, it is sufficient to find $g_{i, j, k}$ in the following relation

$$
\phi_{i}(x) \phi_{j}(x) \simeq \sum_{k=0}^{i+j} g_{i, j, k} \phi_{k}(x)
$$

which is called the linearization formula [34]. Operational matrices are used in several areas of numerical analysis and they hold particular importance in various subjects such as integral equations [36], differential equations [37], integro-differential equations [38] and etc. Also many textbooks and papers have employed the operational matrices for spectral methods. Now we present the following theorem.

Theorem 3. If we consider the Jacobi approximation

$$
y(x) \cong \sum_{i=0}^{m} a_{i} P_{i}^{(\alpha, \beta)}(x)=\left(P^{(\alpha, \beta)}(x)\right)^{T} A,
$$

then

$$
x^{i} y^{(j)}(x) \cong B^{T} P^{(\alpha, \beta)}(x)=\left(\left(G^{i} D^{j}\right)^{T} A\right)^{T} P^{(\alpha, \beta)}(x)
$$

where

$$
D=\left(\begin{array}{cc}
0 & E^{-1} \\
0 & 0
\end{array}\right)_{(n+1) \times(n+1)}, E_{p, q}= \begin{cases}\frac{-2(p+\alpha+1)(p+\beta+1)}{(\alpha+\beta+p+1)(\alpha+\beta+2 p+2)(\alpha+\beta+2 p+3)}, & p=q-2, \\
\frac{2(\alpha-\beta)}{(\alpha+\beta+2 p)(\alpha+\beta+2 p+2)}, & p=q-1, \\
\frac{2(\alpha+\beta+p)}{(\alpha+\beta+2 p-1)(\alpha+\beta+2 p)}, & p=q, \\
0, & \text { otherwise }\end{cases}
$$

and

$$
G_{p, q}= \begin{cases}\frac{2(p+\alpha)(p+\beta)}{(\alpha+\beta+2 p)(\alpha+\beta+2 p+1)}, & p=q-1 \\ \frac{\beta^{2}-\alpha^{2}}{(\alpha+\beta+2 p+1)(\alpha+\beta+2 p+2)}, & p=q \\ \frac{2 p(\alpha+\beta+p)}{(\alpha+\beta+2 p)(\alpha+\beta+2 p-1)}, & p=q+1 \\ 0, & \text { otherwise }\end{cases}
$$

Proof: see [35]. 
Now by using theorem 1, we obtain the operational matrix for the following operator (the two dimensional case)

$$
L(f(x, t))=x^{i} y^{j} \frac{\partial}{\partial x^{k} \partial y^{d}}\left(\sum_{t=0}^{m} \sum_{p=0}^{n} a_{t, p} P_{t}^{(\alpha, \beta)}(x) P_{p}^{(\alpha, \beta)}(y)\right) .
$$

For this goal, we present the following theorem.

Theorem 4. If we consider the Jacobi approximation (39) and the operator (43), then we have

$$
\begin{aligned}
& x^{i} y^{j} \frac{\partial^{k+d}}{\partial x^{k} \partial y^{d}}\left(\sum_{t=0}^{m} \sum_{p=0}^{n} a_{t, p} P_{t}^{(\alpha, \beta)}(x) P_{p}^{(\alpha, \beta)}(y)\right) \simeq\left(P^{(\alpha, \beta)}(x)\right)^{T}\left(\left(D^{k}\right)^{T}\left(G^{i}\right)^{T} A G^{j} D^{d}\right)\left(P^{(\alpha, \beta)}(y)\right)= \\
& \left(P^{(\alpha, \beta)}(x)\right)^{T}\left(A_{(k, d)}^{(i, j)}\right)\left(P^{(\alpha, \beta)}(y)\right) .
\end{aligned}
$$

Proof. If we consider the following relations

$$
\begin{aligned}
& x^{i} y^{j} \frac{\partial^{k+d}}{\partial x^{k} \partial y^{d}}\left(\sum_{t=0}^{m} \sum_{p=0}^{n} a_{t, p} P_{t}^{(\alpha, \beta)}(x) P_{p}^{(\alpha, \beta)}(y)\right)=x^{i} y^{j} \frac{\partial}{\partial x^{k} \partial y^{d}}\left(\left(P^{(\alpha, \beta)}(x)\right)^{T}(C)\left(P^{(\alpha, \beta)}(y)\right)\right)= \\
& x^{i} \frac{\partial^{k}}{\partial x^{k}}\left(P^{(\alpha, \beta)}(x)\right)^{T}(C) y^{j} \frac{\partial d}{\partial y^{d}}\left(P^{(\alpha, \beta)}(y)\right)=\left(P^{(\alpha, \beta)}(x)\right)^{T}\left(\left(D^{k}\right)^{T}\left(G^{i}\right)^{T} A G^{j} D^{d}\right)\left(P^{(\alpha, \beta)}(y)\right)= \\
& \left(P^{(\alpha, \beta)}(x)\right)^{T}\left(A_{(k, d)}^{(i, j)}\right)\left(P^{(\alpha, \beta)}(y)\right),
\end{aligned}
$$

the proof is completed.

Now we present a useful theorem which we need for obtaining the operational matrix with respect to the shift operator. This is also a consequence of the connection formula stated in theorem 1 as well as formula (34).

Theorem 5. If $c \in R$ and $\alpha, \beta>0$, then

$$
\left[P_{0}^{(\alpha, \beta)}(x+c), P_{1}^{(\alpha, \beta)}(x+c), \ldots, P_{n}^{(\alpha, \beta)}(x+c)\right]^{T}=W_{c} K^{-1}\left[P_{0}^{(\alpha, \beta)}(x), P_{1}^{(\alpha, \beta)}(x), \ldots, P_{n}^{(\alpha, \beta)}(x)\right]^{T},
$$

where $W_{c}$ is a lower triangular matrix

$$
\left(W_{c}\right)_{i, j}= \begin{cases}0, & i<j \\ D_{i}^{(\alpha, \beta, j)}, & i \geq j\end{cases}
$$

and

$$
D_{i}^{(\alpha, \beta, j)}=\sum_{k=i}^{j} G_{k}^{(\alpha, \beta, j)} c^{k}\left(\begin{array}{l}
k \\
i
\end{array}\right)
$$

and $G_{k}^{(\alpha, \beta, j)}$ is defined in (25).

Proof: From (19), we have

$$
P_{n}^{(\alpha, \beta)}(x+c)=\sum_{i=0}^{n} G_{i}^{(\alpha, \beta, n)}(x+c)^{i}=\sum_{i=0}^{n} G_{i}^{(\alpha, \beta, n)} \sum_{j=0}^{i}\left(\begin{array}{l}
i \\
j
\end{array}\right) c^{i-j} x^{j},
$$

so if we define

$$
D_{i}^{(\alpha, \beta, j)}=\sum_{k=i}^{j} G_{k}^{(\alpha, \beta, j)} c^{k}\left(\begin{array}{c}
k \\
i
\end{array}\right)
$$

therefore we have

$$
P_{n}^{(\alpha, \beta)}(x+c)=\sum_{i=0}^{n} D_{i}^{(\alpha, \beta, n)} x^{i}
$$

Using obtained result (51), we have

$$
\left[P_{0}^{(\alpha, \beta)}(x+c), P_{1}^{(\alpha, \beta)}(x+c), \ldots, P_{n}^{(\alpha, \beta)}(x+c)\right]^{T}=W_{c}\left[1, x, \ldots, x^{n}\right]^{T}, \forall \alpha, \beta>0,
$$

where $W_{c}$ is given in (47), and using formula (34), we obtain

$$
\left[P_{0}^{(\alpha, \beta)}(x+c), P_{1}^{(\alpha, \beta)}(x+c), \ldots, P_{n}^{(\alpha, \beta)}(x+c)\right]^{T}=W_{c} K^{-1}\left[P_{0}^{(\alpha, \beta)}(x), P_{1}^{(\alpha, \beta)}(x), \ldots, P_{n}^{(\alpha, \beta)}(x)\right]^{T},
$$

therefore $W_{c} K^{-1}$ is the shift operational matrix and the proof of theorem is completed. 
Now from theorems 3 and 5, we can obtain the modified version of theorem 3, for the shifted Jacobi polynomials as

$$
x^{i} y^{(j)}(x+c) \cong B^{T} P^{(\alpha, \beta)}(x)=\left(\left(G^{i} D^{j}\right)^{T} A\right)^{T} W_{c} K^{-1} P^{(\alpha, \beta)}(x), \forall \alpha, \beta>0 .
$$

Finally, combining Eq.(44) and Eq.(54) we conclude

$$
x^{i} y^{j} \frac{\partial^{k+d}}{\partial x^{k} \partial y^{d}}\left(\sum_{t=0}^{m} \sum_{p=0}^{n} a_{t, p} P_{t}^{(\alpha, \beta)}(x) P_{p}^{(\alpha, \beta)}(y)\right)=\left(P^{(\alpha, \beta)}(x+k)\right)\left(\left(M_{h}^{T}\right) A_{(k, d)}^{i, j} M_{k}\right)\left(P^{(\alpha, \beta)}(y+h)\right)^{T}
$$

where from Eq. $(44), A_{(k, d)}^{(i, j)}$

$$
A_{(k, d)}^{(i, j)}=\left(\left(D^{k}\right)^{T}\left(G^{i}\right)^{T} A G^{j} D^{d}\right)
$$

and $M_{h}$ is defined as

$$
M_{h}=W_{c} K^{-1}
$$

\section{The method of solution}

In this section, we describe our new approach for solving the second order linear PDDEs with variable coefficients (1). Our approach is based on approximating the exact solution of Eq.(1) by truncating two dimensional shifted Jacobi expansion as

$$
y(x+h, y+k) \simeq \sum_{i=0}^{m} \sum_{j=0}^{m} a_{i, j} P_{i}^{(\alpha, \beta)}(x+h) P_{j}^{(\alpha, \beta)}(y+k)=\left(P^{(\alpha, \beta)}(x+h)\right)^{T} A\left(P^{(\alpha, \beta)}(y+k)\right),
$$

where

$$
A=\left(\begin{array}{ccc}
a_{11} & \cdots & a_{1 m} \\
\vdots & \ddots & \vdots \\
a_{m 1} & \cdots & a_{m m}
\end{array}\right)
$$

and

$$
P^{(\alpha, \beta)}(x+h)=\left[P_{0}^{(\alpha, \beta)}(x+h), P_{1}^{(\alpha, \beta)}(x+h), \ldots, P_{m}^{(\alpha, \beta)}(x+h)\right]^{T} .
$$

Also we assume that the coefficients $P_{r}(x, y)$ have the two dimensional Taylor series in the following form

$$
P_{r}(x, y)=\sum_{i=0}^{n} \sum_{j=0}^{n} e_{i, j}^{r} x^{i} y^{j}
$$

Now by substituting Eqs.(58) and (61) into Eq.(2), we obtain

$$
\begin{aligned}
& \sum_{i=0}^{n} \sum_{j=0}^{n} e_{i, j}^{0} x^{i} y^{j} \frac{\partial^{2} u}{\partial x^{2}}(x, y)+\sum_{i=0}^{n} \sum_{j=0}^{n} e_{i, j}^{1} x^{i} y^{j} \frac{\partial^{2} u}{\partial x \partial y}(x, y)+\sum_{i=0}^{n} \sum_{j=0}^{n} e_{i, j}^{2} x^{i} y^{j} \frac{\partial^{2} u}{\partial y^{2}}(x, y)+ \\
& \sum_{i=0}^{n} \sum_{j=0}^{n} e_{i, j}^{3} x^{i} y^{j} \frac{\partial u}{\partial x}(x, y)+\sum_{i=0}^{n} \sum_{j=0}^{n} e_{i, j}^{4} x^{i} y^{j} \frac{\partial u}{\partial y}(x, y)+\sum_{i=0}^{n} \sum_{j=0}^{n} e_{i, j}^{5} x^{i} y^{j} u(x, y) \simeq g(x, y),
\end{aligned}
$$

so from Eq.(62), we must simplify the following formula

$$
x^{i} y^{j} \frac{\partial}{\partial x^{k} \partial y^{d}}\left(\sum_{t=0}^{m} \sum_{p=0}^{n} a_{t, p} P_{t}^{(\alpha, \beta)}(x+h) P_{p}^{(\alpha, \beta)}(y+k)\right)=\left(P^{(\alpha, \beta)}(x)\right)\left(\left(M_{h}\right)^{T} A_{(k, d)}^{(i, j)}\left(M_{k}\right)\right)\left(P^{(\alpha, \beta)}(y)\right)^{T}
$$

where $D$ and $G$ are defined in Eqs.(41) and (42) respectively. Also we approximate the right hand side of Eq.(1) as

$$
g(x, y) \simeq \sum_{i=0}^{n} \sum_{j=0}^{n} b_{i, j} P_{i}^{(\alpha, \beta)}(x) P_{i}^{(\alpha, \beta)}(y)=\left(P^{(\alpha, \beta)}(x)\right)^{T} B\left(P^{(\alpha, \beta)}(y)\right)
$$

where

$$
B=\left(\begin{array}{ccc}
b_{11} & \ldots & b_{1 m} \\
\vdots & \ddots & \vdots \\
b_{m 1} & \cdots & b_{m m}
\end{array}\right)
$$

If we define

$$
\left(M_{h}\right)^{T} A_{(k, d)}^{(i, j)}\left(M_{k}=A_{(k, d)}^{(i, j, h, k)}\right.
$$


Then, using Eqs.(63), (66) and (64) into Eq.(62), we obtain

$$
\begin{aligned}
& \left(P^{(\alpha, \beta)}(x)\right)^{T}\left(\sum_{i=0}^{n} \sum_{j=0}^{n} e_{i, j}^{0} A_{(2,0)}^{(i, j, h, k)}+\sum_{i=0}^{n} \sum_{j=0}^{n} e_{i, j}^{1} A_{(1,1)}^{(i, j, h, k)}+\sum_{i=0}^{n} \sum_{j=0}^{n} e_{i, j}^{2} A_{(0,2)}^{(i, j, h, k)}+\sum_{i=0}^{n} \sum_{j=0}^{n} e_{i, j}^{3} A_{(1,0)}^{(i, j, h, k)}+\right. \\
& \left.\sum_{i=0}^{n} \sum_{j=0}^{n} e_{i, j}^{4} A_{(0,1)}^{(i, j, h, k)}+\sum_{i=0}^{n} \sum_{j=0}^{n} e_{i, j}^{5} A_{(0,0)}^{(i, j, h, k)}\right)\left(P^{(\alpha, \beta)}(y)\right) \simeq\left(P^{(\alpha, \beta)}(x)\right)^{T} B\left(P^{(\alpha, \beta)}(y)\right) .
\end{aligned}
$$

From linear independency of the Jacobi polynomials, we conclude that

$$
F=B
$$

where

$$
F=\left(\begin{array}{ccc}
f_{11} & \cdots & f_{1 m} \\
\vdots & \ddots & \vdots \\
f_{m 1} & \cdots & f_{m m}
\end{array}\right)
$$

Therefore from Eq.(68), we have a system of $(m+1) \times(m+1)$ algebraic equations for the $(m+1) \times(m+1)$ unknown coefficients $a_{i, j}$.

\section{Approximations by Jacobi polynomials}

Now in this section, we present some useful theorems which show the approximations of functions by Jacobi polynomials.For this purpose, let us define $\Lambda=\{x \mid-1 \leq x \leq 1\}$ and

$$
J_{N}^{(\alpha, \beta)}=\operatorname{span}\left\{P_{0}^{(\alpha, \beta)}(x), P_{1}^{(\alpha, \beta)}(x), \ldots, P_{N-1}^{(\alpha, \beta)}(x)\right\} .
$$

The $L_{w(\alpha, \beta)}^{2}(\Lambda)$ - orthogonal projection $\pi_{N}^{(\alpha, \beta)}: L^{2}(\Lambda) \rightarrow J_{N}^{(\alpha, \beta)}$ is a mapping in a way that for any $y(x) \in L^{2}(\Lambda)$, we have:

$$
\left\langle\pi_{N}^{(\alpha, \beta)}(y)-y, \Phi\right\rangle=0, \quad \forall \Phi \in J_{N}^{(\alpha, \beta)} .
$$

Due to the orthogonality, we can write

$$
\pi_{N}^{(\alpha, \beta)}(y)=\sum_{k=0}^{N-1} c_{k} P_{k}^{(\alpha, \beta)}(x)
$$

where $c_{i}(i=0,1, \ldots, N-1)$ are constants in the following form

$$
c_{i}=\frac{1}{\gamma_{k}}<y(x), P_{k}^{(\alpha, \beta)}>_{L_{w}^{2}(\alpha, \beta)} .
$$

In the literature of spectral methods, $\pi_{N}^{(\alpha, \beta)}(y)$ is named as Jacobi spectral expansion of $y(x)$ and approximates $y(x)$ on $[-1,1]$. In the spectral methods, by substituting the Jacobi spectral expansion $\pi_{N}^{(\alpha, \beta)}(y)$ in the ODEs and their boundary conditions, we obtain a residual term which is symbolically showed by $\operatorname{Res}(x)$ as a function of $x$ and $N, \alpha, \beta$. Different strategies for minimizing a residual term $\operatorname{Res}(x)$, lead to different versions of spectral methods such as Galerkin, Tau and collocation methods. For instance, in the collocation methods the residual term $\operatorname{Res}(x)$ is vanished in particular points named as collocated points. Also estimating the distance between $y(x)$ and its Jacobi spectral expansion as measured in the weighted norm $\|\cdot\|_{w^{(\alpha, \beta)}}$ is an important problem in numerical analysis. The following theorems provide the basic approximation results for Jacobi spectral expansion.

Theorem 6. we have

$$
\begin{gathered}
\left\|\frac{d^{l}}{d x^{l}}\left(\pi_{N}^{(\alpha, \beta)}(y)-y\right)\right\|_{w^{(\alpha+l, \beta+l)}} \leq N^{l-m}\left\|\frac{d^{m}}{d x^{m}} y(x)\right\|_{w^{(\alpha+m, \beta+m)}}, \\
0 \leq l \leq m, \quad \forall y \in B_{(\alpha, \beta)}^{m}(\Lambda)
\end{gathered}
$$

where

$$
B_{(\alpha, \beta)}^{m}(\Lambda)=\left\{\forall y \in L_{w^{(\alpha, \beta)}}^{2}: \frac{d^{l} y}{d x^{l}} \in L_{w^{(\alpha+l, \beta+l)}}^{2}(\Lambda), \quad 0 \leq l \leq m\right\}
$$

Proof: See [31]. 
Definition 5. The Sturm-Liouville operator $\mathrm{E}$ on the interval $-1<x<1$, i.e., is of the form

$$
\mathrm{\iota}(y)=-\left(p(x) y^{\prime}\right)^{\prime}+q(x) y=\lambda w(x) y,
$$

with $y^{\prime}( \pm 1)$ bounded, where $p(x)>0, q(x) \geq 0$ and $w(x) \geq 0$ are continuously differentiable functions on the open interval $(-1,1)$ and $p( \pm 1)=0$.

Theorem 7. Let $y(x) \in L_{w(\alpha, \beta)}^{2}[-1,1]$, and

$$
\pi_{N}^{(\alpha, \beta)}(y)=\sum_{k=0}^{N-1} c_{k} P_{k}^{(\alpha, \beta)}(x)
$$

then

and

$$
\left|c_{k}\right| \simeq \frac{C}{\left(\lambda_{k}\right)^{m}}\left\|y_{(m)}\right\|_{w^{(\alpha, \beta)}}
$$

$$
\left\|y(x)-\sum_{k=0}^{N-1} c_{k} P_{k}^{(\alpha, \beta)}(x)\right\|_{w^{(\alpha, \beta)}}^{2}=\sum_{k=N}^{\infty} \gamma_{k} c_{k}^{2}
$$

where

$$
\begin{gathered}
\lambda_{k}=k(k+\alpha+\beta+1), \\
c_{k}=\frac{1}{\gamma_{k}}<y(x), P_{k}^{(\alpha, \beta)}>_{L_{w}^{2}(\alpha, \beta)}, \quad \gamma_{k}=\left\|\left(P_{k}^{(\alpha, \beta)}\right)^{2}\right\|_{L_{w}^{2}(\alpha, \beta)} .
\end{gathered}
$$

$C$ is a constant and $m$ is chosen in a way that $y^{(2 m)} \in L_{w^{(\alpha, \beta)}}^{2}[-1,1]$.

Also we have the symbol

$$
y_{(m)}=\frac{1}{w(x)} \mathrm{Ł} y_{m-1}(x)=\left(\frac{£}{w(x)}\right)^{m} y(x),
$$

and $y_{(0)}(x)=y(x)$.

Proof: See [32].

Theorem 5, shows that under particular conditions the eigen-solutions of the singular Sturm-Liouville problems in finite interval (Jacobi polynomials) are well suited for expanding arbitrary functions defined in the finite interval and convergence of any function and its Jacobi spectral expansion solely depend on the power decay of the Jacobi spectral expansion coefficients. Consequently from theorem 5 , we conclude that if the function $y(x) \in C^{\infty}[-1,1]$, we recover spectral decay of the expansion coefficients. i.e., $\left|c_{k}\right|$ decays faster than any algebraic order of $\lambda_{k}$. This result is valid and independent of specific boundary conditions on $y(x)$ [32].

\section{The test experiments}

In this section, two numerical experiments are given to illustrate the properties of the method and all of them were performed on the computer using a program written in Matlab 2013. To measure the accuracy, the following error functions are defined:

$$
\begin{aligned}
& \text { M. error }=\max _{i, j=1, \ldots, n}\left|u(i, j)-u_{\text {exact }}(i, j)\right| \\
& \text { Relative. error }=\sqrt{\frac{\sum_{j=1}^{n}\left(u(i, j)-u_{\text {exact }}(i, j)\right)^{2}}{\sum_{j=1}^{n}\left(u_{\text {exact }}(i, j)\right)^{2}}} \\
& \text { Me.error }=\frac{1}{(m+1)(n+1)} \sum_{i=1}^{m} \sum_{j=1}^{n}\left|u(i, j)-u_{\text {exact }}(i, j)\right| .
\end{aligned}
$$

Experiment 1. Consider the second-order initial value PDE [17]

$$
u_{t t}=u_{x x}+6, \quad u(x, 0)=x^{2}, \quad u_{t}(x, 0)=4 x .
$$

The exact solution is $u(x, t)=(x+2 t)^{2}$.

Now we approximate the exact solution of Eq.(74) by

$$
u(x, t) \simeq \sum_{i=0}^{m} \sum_{j=0}^{m} c_{i, j} P_{i}^{(\alpha, \beta)}(x) P_{j}^{(\alpha, \beta)}(y)=\left(P^{(\alpha, \beta)}(x)\right)^{T} C\left(P^{(\alpha, \beta)}(y)\right),
$$


where the shifted Jacobi coefficients matrix $C$ is given by

$$
C=\left(\begin{array}{ccc}
c_{11} & \ldots & c_{1 m} \\
\vdots & \ddots & \vdots \\
c_{m 1} & \cdots & c_{m m}
\end{array}\right)
$$

The matrix form of the Eq.(74) is

$$
\left(P^{(\alpha, \beta)}(x)\right)^{T}\left(\left(D^{2}\right)^{T} C-C D^{2}\right)\left(P^{(\alpha, \beta)}(y)\right)=B,
$$

where $B$ is a triangular matrix in the following form

$$
B_{i, j}= \begin{cases}6 \eta_{i}^{(\alpha, \beta)}, & i=j \\ 0, & i \neq j\end{cases}
$$

By implementing our method as presented in section 4 , for $n=5$, we obtain the exact solution.

Experiment 2. Consider the second-order PDE with variable coefficients [17]

$$
x^{2} u_{x x}-y^{2} u_{y y}=0
$$

subject to the conditions

$$
\begin{aligned}
& u(0, y)=1, \quad u(1, y)=e^{y} \\
& u(x, 0)=1, \quad u(x, 1)=e^{x}
\end{aligned}
$$

Now we approximate the exact solution of Eq.(79) by

$$
u(x, t) \simeq \sum_{i=0}^{m} \sum_{j=0}^{m} c_{i, j} P_{i}^{(\alpha, \beta)}(x) P_{j}^{(\alpha, \beta)}(y)=\left(P^{(\alpha, \beta)}(x)\right)^{T} C\left(P^{(\alpha, \beta)}(y)\right)
$$

where the shifted Jacobi coefficients matrix $C$ is given by

$$
C=\left(\begin{array}{ccc}
c_{11} & \ldots & c_{1 m} \\
\vdots & \ddots & \vdots \\
c_{m 1} & \cdots & c_{m m}
\end{array}\right)
$$

The matrix form of Eq.(79) is

$$
\left(P^{(\alpha, \beta)}(x)\right)^{T}\left(\left(D^{2}\right)^{T}\left(G^{2}\right)^{T} C-C D^{2} G^{2}\right)\left(P^{(\alpha, \beta)}(y)\right)=0 .
$$

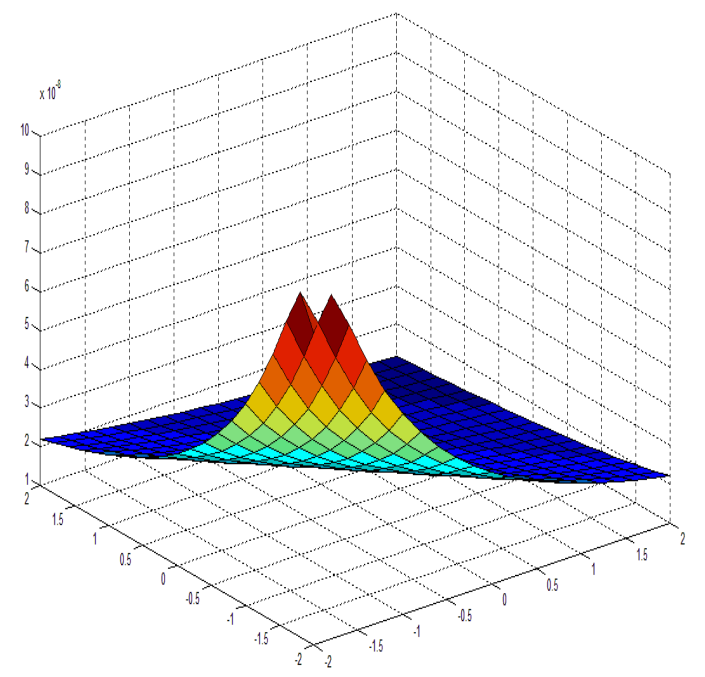

Figure 1. The M-error function for $\alpha=0.5, \beta=0.5, m=8$ of experiment 2

By implementing our method as presented in section 4 , for $n=5$, we obtain

$$
u(x, y)=1+x y+\frac{(x y)^{2}}{2}+\frac{(x y)^{3}}{3}+\frac{(x y)^{4}}{4}
$$




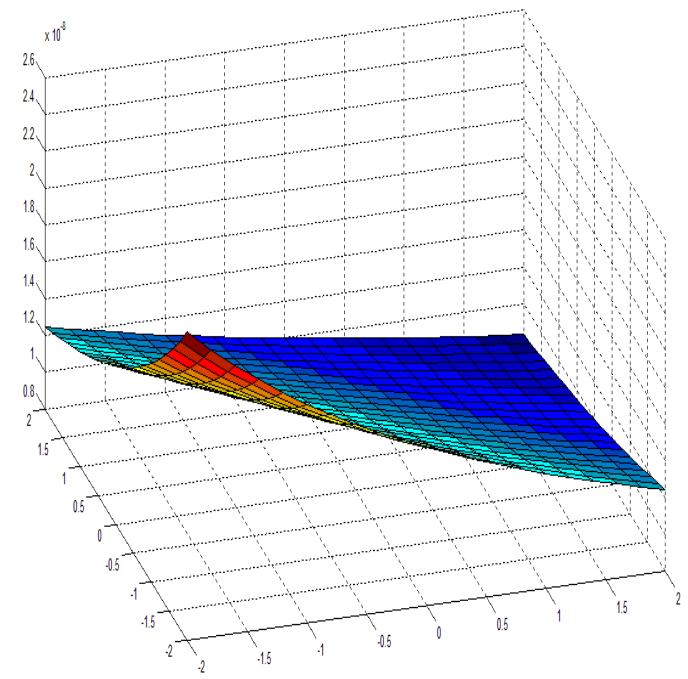

Figure 2. The M-error function for $\alpha=0.5, \beta=0.5, m=15$ of experiment 2

which is the first five terms of the two dimensional Taylor series of $e^{(x y)}$.

We have observed that this method (the general shifted Jacobi matrix method) is very efficient for numerical approximation of the general high order linear PDEs with variable coefficients. Also, during the running of programs, we found out the run time of the general shifted Jacobi matrix scheme is 2.015 second and the run time of the collocation method and Taylor approximation is 10.047 and 11.018 seconds, respectively. Thus in the case of the numerical solution of linear PDEs with variable coefficients, we prefer the general shifted Jacobi matrix scheme to the collocation method and Taylor approximation. This is the excellent advantage on the application of the general shifted Jacobi matrix scheme to the linear PDEs with variable coefficients. Also, closer look at the results of the general shifted Jacobi matrix scheme reveals that this method of solution is also stable.

\section{Application of the method for the high order linear PDDEs}

In this section, we report the numerical results obtained for a high order linear PDDE by the abovementioned procedure. This shows that it is straightforward to extend the method to the high order linear PDDEs as follows.

Experiment 3. Consider the fifth order linear PDDE [39]

$$
\frac{\partial^{5} u}{\partial x^{5}}+x^{2} \frac{\partial^{3} u}{\partial y^{3}}+3 x^{5} \frac{\partial^{2} u}{\partial x \partial y}+12 u=720 x+12 y^{4}+12 x^{3},
$$

subject to the conditions

$$
\begin{aligned}
& u(0, y)=y^{4}, \quad \frac{\partial^{4} u}{\partial x^{4}}(0, y)+u(1, y)-u(-1, y)=2, \quad \frac{\partial^{3} u}{\partial x^{3}}(1, y)+y \frac{\partial u}{\partial x}(0, y)=126, \\
& \frac{\partial^{2} u}{\partial x \partial y}(x, 1 / 2)-\frac{\partial u}{\partial y}(x, 0)=2 x^{2}-4 x, \quad 3 x^{3} \frac{\partial u}{\partial y}(x, 1)+\frac{\partial u}{\partial x}(x,-1)=12 x^{3}+3 x^{2}+4 x .
\end{aligned}
$$

The exact solution of this equation is

$$
u(x, y)=x^{6}+y^{4}-2 x^{2} y+x^{3} .
$$

By implementing the method as presented in section 4 , for $m=5$ and $m=8$ and also for different parameters of $\alpha$ and $\beta$, we obtain the approximate solutions. The Relative-error functions of this problem, are shown in figures 3 and 4. Also, tables 1 and 2 represent a comparison of the results of the present method with ref. [39].

Table 1. The Me.error for the method of [39] of experiment 3

\begin{tabular}{|c|c|c|c|c|c|c|}
\hline \multicolumn{7}{|c|}{$\mathrm{n}$} \\
\hline $\mathrm{m}$ & 4 & 5 & 6 & 7 & 8 & 9 \\
\hline 6 & $3.38 \times 10^{-12}$ & $1.38 \times 10^{-11}$ & $1.55 \times 10^{-11}$ & $3.08 \times 10^{-11}$ & $5.53 \times 10^{-10}$ & $2.86 \times 10^{-9}$ \\
\hline 7 & $2.11 \times 10^{-12}$ & $2.8 \times 10^{-12}$ & $1.05 \times 10^{-11}$ & $1.22 \times 10^{-9}$ & $7.43 \times 10^{-11}$ & $5.87 \times 10^{-11}$ \\
\hline 8 & $1.25 \times 10^{-11}$ & $9.66 \times 10^{-12}$ & $3.17 \times 10^{-11}$ & $4.14 \times 10^{-11}$ & $2.23 \times 10^{-7}$ & $1.12 \times 10^{-8}$ \\
\hline 9 & $1.12 \times 10^{-10}$ & $9.88 \times 10^{-11}$ & $1.35 \times 10^{-10}$ & $9.91 \times 10^{-11}$ & $5.41 \times 10^{-10}$ & $1.64 \times 10^{-7}$ \\
\hline
\end{tabular}




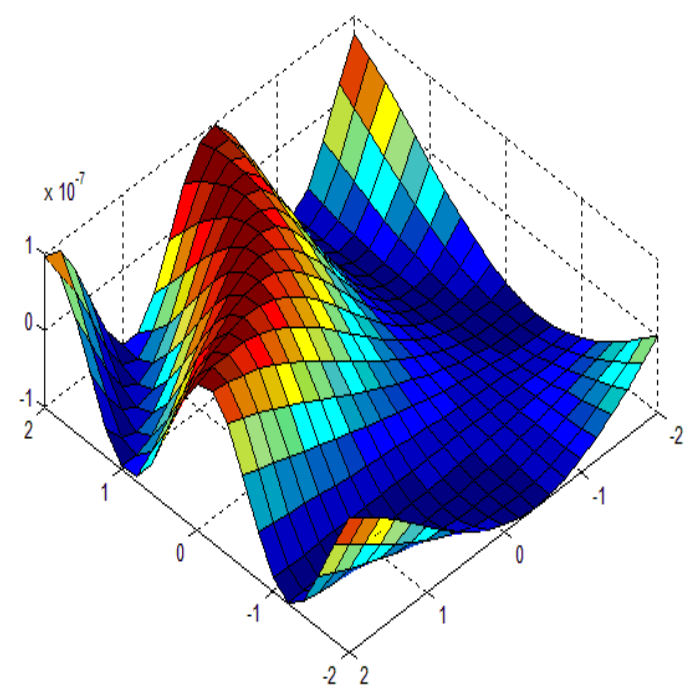

Figure 3. The Relative-error function for $\alpha=0.5, \beta=0.5, m=5$, of experiment 3

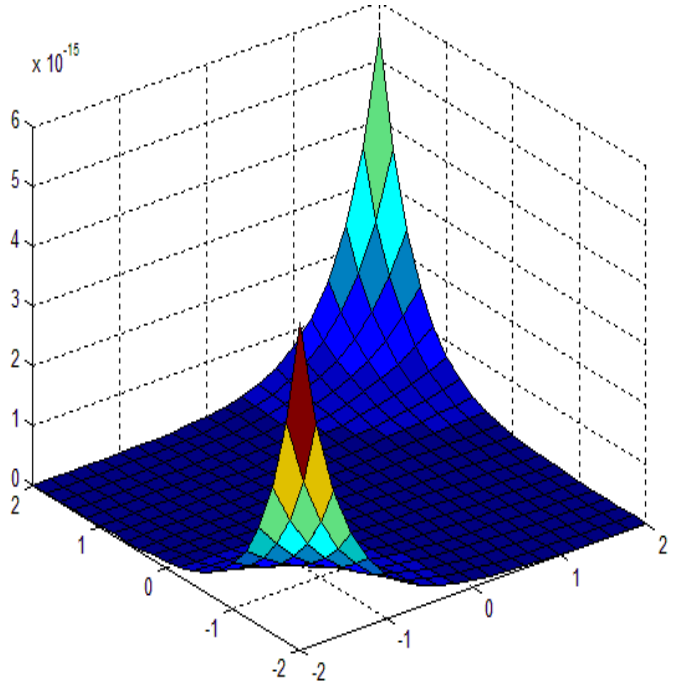

Figure 4. The Relative-error function for $\alpha=2, \beta=2, m=8$, of experiment 3 
The General Two Dimensional Shifted Jacobi Matrix Method for Solving the

Table 2. The Me.error for the present method for $\alpha=0.4, \beta=0.8$ of experiment 3

\begin{tabular}{|c|c|c|c|c|c|c|}
\hline \multicolumn{7}{|c|}{$\mathrm{n}$} \\
\hline $\mathrm{m}$ & 4 & 5 & 6 & 7 & 8 & 9 \\
\hline 6 & $2.46 \times 10^{-12}$ & $0.33 \times 10^{-12}$ & $3.77 \times 10^{-12}$ & $2.57 \times 10^{-12}$ & $3.89 \times 10^{-11}$ & $1.73 \times 10^{-10}$ \\
\hline 7 & $1.29 \times 10^{-12}$ & $1.18 \times 10^{-13}$ & $4.25 \times 10^{-12}$ & $0.17 \times 10^{-9}$ & $4.99 \times 10^{-11}$ & $3.39 \times 10^{-12}$ \\
\hline 8 & $1.09 \times 10^{-11}$ & $8.64 \times 10^{-13}$ & $1.61 \times 10^{-12}$ & $3.88 \times 10^{-11}$ & $1.22 \times 10^{-7}$ & $1.01 \times 10^{-9}$ \\
\hline 9 & $0.44 \times 10^{-10}$ & $7.39 \times 10^{-12}$ & $1.22 \times 10^{-11}$ & $7.17 \times 10^{-11}$ & $5.11 \times 10^{-10}$ & $1.32 \times 10^{-8}$ \\
\hline
\end{tabular}

\section{Conclution}

In this paper, we have introduced a new and efficient approach for numerical approximation of the second order linear PDDEs with variable coefficients. The method is based on the approximation of an exact solution with the two dimensional shifted Jacobi polynomial approximation with variable coefficients in the two dimensional Taylor series. Implementation of the method reduces the problem to a system of algebraic equations. Hence, the applicability of the present work is in considering of the general second order linear PDDE (1), whereas the other papers monitor only the particular cases of our general study. Also, using the general shifted Jacobi polynomials as the basis functions for numerical approximation wherein the shifted Chebyshev and Legendre polynomials are the particular cases of them, is the other superiority of the present study. Furthermore, the text experiments represent the accuracy and the efficiency of the method with only few terms in the expansion of Taylor series.

\section{Acknowledgements}

We are grateful to the reviewers and the editor for their helpful comments and suggestions which indeed improved the quality of this manuscript.

\section{REFERENCES}

[1] R. Agarwal, D. ORegan, Ordinary and Partial Differential Equations, Springer, 2009.

[2] L.C. Evans, Partial Differential Equations, American Mathematical Society, 1999.

[3] W.E. Schiesser, G.W. Griffiths, A compendium of partial differential equation models (method of Lines Analysis with Matlab), Cambridge University, 2009.

[4] J. Geiser, Decomposition methods for differential equations( Theory and Applications), Taylor and Francis, 2009.

[5] A.M. Wazwaz, Partial Differential Equations and Solitary Waves Theory, Springer, 2009.

[6] R. Camassa, D. Holm, An integrable shallow water equation with peaked solitons, Phys. Rev. Lett, vol. 71, pp. 16611664, 1993.

[7] A. Degasperis, M. Procesi, Asymptotic Integrability, Symmetry and Perturbation Theory, World Scientific, vol. 20, pp. 23-37, 1999.

[8] V. Eguiluz, E. Hernandez-Garcia and O. Piro, Boundary effects in the complex Ginzburg- Landau equation, Internat. J. Bifur. Chaos, vol. 23, pp. 2209-2214, 1999.

[9] B. Fuchssteiner, Some tricks from the symmetry-toolbox for nonlinear equations: Generalization of the Camassa-Holm equation, Physica D, vol. 95, pp. 229-243, 1996.

[10] B. Fuchssteiner and A. Fokas, Sympletic structures, their Backlund transformations and hereditary symmetries, Physica D, vol. 4 , pp. 47-66, 1981.

[11] Malfliet W., Solitary wave solutions of nonlinear wave equations, Am. J. Phys, vol. 60, pp. 650- 654, 1992.

[12] H. Holden, K.H. Karlsen, K.A. Lie, N.H. Risebro, Splitting methods for partial differential equations with Rough Solutions (Analysis and MATLAB programs), European Mathematical Society, 2010.

[13] K. W. Morton, D. F. Mayers, Numerical solution of partial differential equations, Cambridge University, 2009.

[14] M.H. Giga, Y. Giga, J. Saal, Nonlinear partial differential equations (Asymptotic Behavior of Solutions and Self-Similar Solutions), Springer, 2010.

[15] A. Quarteroni, A.Valli, Numerical approximation of partial differential equations, Springer, 2008. 
[16] P. Solin, Partial differential equations and the finite element method, John wiley and sons, 2008.

[17] Cenk Kesan, Chebyshev polynomial solutions of second-order linear partial differential equations, Appl Math Comput, vol. 134, pp. 109-124, 2003.

[18] C. Kesan, Taylor polynomial solutions of second order linear partial differential equations, Appl Math Comput, vol. 152, pp. 29-41, 2004.

[19] A.M. Wazwaz, A comparison between the variational iteration method and Adomian decomposition method,Comput Appl Math, vol. 207, pp. 129-136, 2007.

[20] A.M. Wazwaz, S.M. Sayed, A new modification of Adomian decomposition method for linear and nonlinear operators. Appl Math Comput, vol. 122, pp. 393-405, 2011.

[21] S.H. Ho, C.K. Chen, Analysis of general elastically end restrained non-uniform beams using differential transform, Appl Math Model, vol. 22, pp. 219-234, 1998.

[22] N. Bildik, A. Konuralp, F.O. Bek, S. Kucukarslan, Solution of different type of the partial differential equation by differential transform method and Adomian's decomposition method, Appl Math Comput, vol. 172, pp. 551-567, 2006.

[23] Z.M. Odibat, C. Bertelle, M.A. Alaoui, G.H.E. Duchamp, A multi-step differential transform method and application tonon-chaotic or chaotic systems, Comput Math Appl, vol. 59, pp. 1462-1472, 2010.

[24] W. G. Szymczak, I. Babuska, Adaptivity and error estimation for the finite element method applied to convection diffusion problems, SIAM J. Number. Anal, Vol. 21, No. 5, 1984.

[25] I. Babuska, J. E. Osborn, Generalized Finite Element Methods: Their Performance and Their Relation to Mixed Methods, SIAM J. Number. Anal, vol. 20, No. 3, 1983.

[26] I. Babuska, The Finite Element Method with Penalty, Mathematics of Computation, vol. 27, No. $122,1973$.

[27] I. Babuska, The Finite Element Method for Infinite Domains, Mathematics of Computation, vol. 26, No. 117, 1972.

[28] F. Shakeri, M. Dehghan, A finite volume spectral element method for solving magnetohydrodynamic (MHD) equations, Applied Numerical Mathematics, vol. 61, pp. 1-23, 2011.

[29] A. Ahmadian, M. Suleiman, S. Salahshour and D. Baleanu, A Jacobi operational matrix for solving a fuzzy linear fractional differential equation, Advances in Difference Equations, vol. 34, pp. 104-120, 2013.

[30] B. Y. Guo, J. Shen, L. L. Wang, Generalized Jacobi polynomials/functions and their applications, Applied Numerical Mathematics, vol. 59, pp. 1011-1028, 2009.

[31] D. Funaro, Polynomial Approximations of Differential Equations. Springer-Verlag, 1992.

[32] J.S. Hesthaven, S. Gottlieb, D. Gottlieb, Spectral Methods for Time-Dependent Problems, first ed, Cambridge University, 2007.

[33] A. Imani, A. Aminataei, A. Imani, Collocation method via Jacobi polynomials for solving nonlinear ordinary differential equations, Int. J. Math. Math. Sci., Article ID 673085, 11P, 2011.

[34] M. R. Eslahchi, M. Dehghan, Application of Taylor series in obtaining orthogonal operational matrix, J. Comput. Math. Appl, vol. 61, pp. 2596-2604, 2011.

[35] M.R. Eslahchi, M. Dehghan, S. Ahmadi-Asl, The general Jacobi matrix method for solving some nonlinear ordinary differential equations, Appl. Math. Model, vol. 36, pp. 3387-3398, 2012.

[36] M. Razzaghi, S. Yousefi, Legendre wavelets method for the nonlinear Volterra- Fredholm integral equations, Math. Comput. Simul, vol. 70, pp. 1-8, 2005.

[37] S.A. Yousefi, M. Behroozifar, Operational matrices of Bernstein polynomials and their applications, Inter Systems Scie, vol. 32, pp. 709-716, 2010.

[38] H. Danfu, S. Xufeng, Numerical solution of integro-differential equations by using CAS wavelet operational matrix of integratio, Appl Math Comput, vol. 194, pp. 460-466, 2007.

[39] A.A. Dascioglu, Chebyshev Polynomial Approximation for High-Order Partial Differential Equations with Complicated Conditions, Numer Methods Partial Differential Eq, Wiley Inter Science, 2008. 